\title{
O EFEITO DA VALERIANA NO TRATAMENTO DA INSÔNIA
}

\section{VALERIANA'S EFFECT ON INSOMNIA TREATMENT}

\author{
SECCHI, Paula1; VIRTUOSO, Suzane²
}

\begin{abstract}
${ }^{1}$ Aluna do Curso de Pós-Graduação em Farmacologia Aplicada da Universidade Estadual do Oeste do Paraná-UNIOESTE, Cascavel, PR; e-mail: paulasecchi@yahoo.com.br

${ }^{2}$ Professora do Curso de Farmácia da Universidade Estadual do Oeste do Paraná - UNIOESTE
\end{abstract}

\section{RESUMO:}

A Valeriana é um dos fitoterápicos mais popularmente utilizados na insônia. Seu uso tem sido relatado desde os tempos da Grécia antiga e Roma. Encontra-se descrita em dispensatórios, compêndios oficiais e farmacopéias. O objetivo deste trabalho foi avaliar a efetividade da Valeriana no tratamento da insônia, por meio de uma revisão de artigos científicos. Utilizou-se o método de revisão sistemática, através da busca de artigos publicados em diversas bases de dados. Não houve restrição quanto à língua das publicações, já que a maioria dos artigos científicos foi publicada em inglês; e nem quanto a data das publicações, uma vez que existem relatos antigos que comprovam a eficácia da Valeriana no tratamento da insônia. Foram excluídos estudos não realizados em seres humanos e estudos que utilizaram preparações combinadas. Selecionaram-se ensaios que compararam a Valeriana com o placebo. Não houve restrição quanto ao emprego de participantes saudáveis ou com distúrbios do sono, e ensaio duplo-cego, randomizado ou cruzado. Foram identificados 110 artigos; entretanto, destes foram eliminados os que não eram ensaios clínicos, estudos realizados em ratos, os que testaram Valeriana com outras ervas ou medicamentos e que não tiveram o sono como desfecho. Somente 15 artigos e 4 revisões sistemáticas foram incluídos na revisão. A maioria dos ensaios clínicos analisados apresentou resultados promissores, mas foram inconclusivos. Devido aos problemas metodológicos e resultados conflitantes apresentados nos ensaios clínicos, as evidências de eficácia disponíveis são fracas para justificar o uso da Valeriana no tratamento da insônia, indicando a necessidade da realização de novos estudos.

Palavras-chave: Valeriana. Valeriana officinalis. Insônia. Plantas medicinais. Distúrbios do sono.

\section{ABSTRACT:}

Valerian is one of the most popular herbal medicine used in insomnia. It's use has been reported since the time of ancient Greece and Rome. It's described in dispensatories, 
official compendiums and pharmacopoeias. The objective of this study was to evaluate the effectiveness of Valerian for insomnia treatment through a scientific article review. The method employed was a systematic review by the search for published articles in many databases. There was no restriction on the language of the publications, since most scientific articles were published in English; neither there was restrictions regarding the publishing date, since past reports have shown proof of effectiveness of Valerian for insomnia treatment. The studies not conducted in humans and studies using combined preparations were all excluded. Trials comparing Valerian with placebo were selected. There was no restriction on the use of healthy or with sleep disorders participants, and double-blind trial, randomized or crossover design. One hundred and ten articles were identified; however, those which were not clinical trials, studies in rats, studies that tested Valerian with other herbs or drugs and studies which had no sleep as the objective were eliminated. Only fifteen articles and four systematic reviews were included in this review. Most of the analyzed clinical trials showed promising results, but somehow inconclusive. Due to methodological problems and conflicting results shown in clinical trials, the available evidence of effectiveness is weak to justify the use of Valerian for insomnia treatment, indicating the need for new studies.

Keywords: Valerian. Valeriana officinalis. Insomnia. Medicinal plants. Sleep disorders.

\section{INTRODUÇÃO}

A insônia é um distúrbio do sono que consiste na incapacidade de iniciar ou manter o sono, ou ter uma duração e qualidade adequada para restaurar a energia e o estado de vigília normal.

É o distúrbio do sono que mais acomete a população e tem sido associado com a diminuição no desempenho do trabalho, o aumento na incidência de acidentes de carro, e maior propensão às doenças (SARRAIS; MANGLANO, 2007).

De acordo com o DSM-IV (Diagnostic and Statistical Manual of Mental Disorders), a característica essencial da insônia é a dificuldade para iniciar ou manter o sono, ou um sono não reparador por pelo menos um mês. Para serem considerados clinicamente significativos, os sintomas devem estar associados com sofrimento importante ou com debilidade social, ocupacional ou outras áreas importantes. Ainda, não deve ocorrer exclusivamente na presença de outros distúrbios do sono, distúrbios mentais, ou efeitos fisiológicos diretos de uma substância ou condições médicas (AMERICAN PSYCHIATRICASSOCIATION, 1994).

Como resultado das diferentes definições de insônia, as estimativas de prevalência de insônia têm variado muito, de 10\% a $40 \%$. Nos primeiros anos de epidemiologia da insônia, aproximadamente há 25 anos, a prevalência da doença era de $40 \%$ da população. Com definições mais restritas em termos de gravidade e freqüência da insônia, a prevalência diminuiu para 15-20\% da população (OHAYON, 2002). De acordo com a declaração de 2005 do Instituto Nacional de Saúde (National Institutes of Health), a insônia tem prevalência de $10 \%$ da população, conforme a definição de ocorrer debilitação durante o dia. Considerando todas as informações 
disponíveis, a prevalência de sintomas de insônia pode ser estimada em 30\% para insônia e 5-10\% para distúrbios específicos (ROTH, 2007).

Cerca de $30 \%$ da população mundial sofre de insônia, sendo mais freqüente em pacientes idosos com ou sem patologia crônica, mas apenas $10 \%$ recebem tratamento adequado (FERNÁNDEZ-SAN-MARTíN et al, 2010). Vários estudos realizados em diversos países ao redor do mundo relataram que aproximadamente $30 \%$ a $40 \%$ dos adultos têm dificuldade de iniciar ou manter o sono. Uma menor porcentagem de adultos relatou problemas graves (10-15\%), mas a prevalência de distúrbios crônicos do sono aumentou para $25 \%$ em idosos (BENT et al, 2006). Insônia também é muito comum em pacientes com problemas médicos crônicos e é encontrada em até $69 \%$ dos pacientes de atenção primária clínica (BENT et al, 2006). Mais de $50 \%$ dos pacientes de atenção primária se queixam de insônia quando são questionados sobre o sono, mas apenas 30\% mencionam este problema ao seu médico por iniciativa própria, e somente $5 \%$ vão ao médico com a finalidade de receber tratamento para este problema (SMITH et al, 2002).

Bastien, Vallières e Morin (2004) demonstraram que a família, trabalho ou escola, e eventos de saúde são os mais comuns fatores desencadeantes de insônia. Associações entre insônia e uma variedade de condições médicas foram constatados por Taylor et al (2007). Indivíduos com hipertensão arterial, dor crônica, dificuldades na respiração, problemas gastrointestinais e urinários se queixavam de insônia com mais freqüência do que os indivíduos com as demais patologias. Além de comorbidades psiquiátricas e médicas, a insônia está associada com importantes conseqüências pessoais e sociais. Hajak et al (2001) demonstraram que uma maior proporção de indivíduos com insônia classificaram sua qualidade de vida como ruim (22\%) quando comparados com indivíduos sem queixas de sono (3\%). Em uma revisão de estudos epidemiológicos, Taylor, Lichstein e Durrence (2003) verificaram que a insônia pode levar à depressão, ansiedade, abuso ou dependência, e ao suicídio.

A Comissão Nacional de Pesquisas de Distúrbios do Sono estimou que os custos diretos de insônia nos Estados Unidos, em 1995, foram de \$13,9 bilhões de dólares (WALSH; ENGELHARDT, 1999), enquanto que Leger, Levy e Paillard (1999), estimaram que o custo total de insônia na França neste mesmo ano foi de aproximadamente $\$ 2$ bilhões.

Desde meados de 1800, uma variedade de medicamentos tornou-se disponível para o tratamento da insônia. O brometo foi o primeiro agente usado como sedativo hipnótico, seguido por hidrato de cloral, paraldeído e uretano. Os barbitúricos foram introduzidos no início de 1900, e em 1960 constituíram cerca de 55\% das prescrições hipnóticas. Em 1970, os diversos benzodiazepínicos tornaram-se disponíveis e rapidamente transformaram-se no tratamento de escolha para a insônia. Nas últimas duas décadas, várias novas classes farmacológicas foram introduzidas e estão 
substituindo os benzodiazepínicos. Os hipnóticos são o tratamento mais comum para a insônia, e seu uso é desproporcionalmente mais elevado em adultos mais velhos (TARIQ; PULISETTY, 2008).

Entre os medicamentos utilizados para o tratamento da insônia, encontram-se os benzodiazepínicos, que são os mais utilizados. Porém, seu uso prolongado produz efeitos adversos como dependência, sedação diurna (ressaca), má qualidade do sono, o que induz a busca por tratamentos alternativos seguros entre eles os produtos fitoterápicos (FERNÁNDEZ-SAN-MARTíN et al, 2010).

Os medicamentos naturais fornecem uma alternativa segura, eficaz e mais econômica para a população, e pesquisas confirmam estes achados. De acordo com uma pesquisa recente, cerca de 1,6 milhões de americanos fazem uso de medicina alternativa e complementar (CAM) para tratamento de distúrbio do sono (PEARSON; JOHNSON; NAHIN, 2006).

A Valeriana é uma planta perene nativa da Europa e da Ásia, e naturalizada na América do Norte. O gênero Valeriana inclui mais de 250 espécies. A espécie de Valeriana mais comumente utilizada na terapêutica é a Valeriana officinalis, apesar de V. edulis (Valeriana mexicana) e V. wallichii (Valeriana indiana) também serem utilizadas. É classificada como "suplemento alimentar", no âmbito de ação do Suplemento Alimentar da Saúde e Educação (NATIONAL INSTITUTES OF HEALTH, 2008).

A composição química da Valeriana varia de acordo com a subespécie, variedade, idade da planta, condições de crescimento, idade e tipo do extrato (WORLD HEALTH ORGANIZATION, 1999). A raiz de Valeriana contém mais de 150 componentes já identificados, entre eles estão os monoterpenos bicíclicos (valpotriatos - valtrato e dihidrovaltrato), óleos voláteis (valeranona, valerenal e ácidos valerênicos), sesquiterpenos, lignanas e alcalóides. Também estão presentes aminoácidos livres, como o gama-aminobutírico (GABA), tirosina, arginina e glutamina (HADLEY; PETRY, 2003). Os valpotriatos estão presentes somente na planta fresca. Podem eventualmente existir em pequenas quantidades se a secagem for realizada em uma temperatura inferior a $40^{\circ} \mathrm{C}$, uma vez que são altamente instáveis e decompõem-se facilmente pelo calor, umidade ou variações de $\mathrm{pH}$, originando outros compostos. Além disso, são insolúveis em água, existindo apenas em preparações não aquosas. A decomposição dos valpotriatos produz pequenas quantidades do ácido isovalérico, que é responsável pelo odor característico desagradável da planta.

Extrações da Valeriana são realizadas através da imersão de raiz seca ou rizoma da planta em solução (água, etanol e água ou metanol e água), seguida de centrifugação e secagem para extrair e concentrar os componentes da planta. Segundo a Farmacopéia Americana de Ervas, a extração de ácido valerênico exige pelo menos $30 \%$ de álcool e a extração de valpotriatros exige $70 \%$ de álcool (HERBALIST, 1999). 
É um dos fitoterápicos mais popularmente utilizado na insônia pelas suas propriedades sedativas e hipnóticas, e menos comumente utilizado como ansiolítico. Algumas preparações têm sido utilizadas para úlcera péptica, gastrite, dispepsia, doenças inflamatórias intestinais crônicas, colo irritável, e também como anticonvulsivante, antidepressiva, antiespasmolítica, antihipertensiva e miorelaxante.

Desde os tempos da Grécia antiga e Roma, a Valeriana tem sido utilizada como medicamento fitoterápico. Seu uso terapêutico foi descrito inicialmente por Hipócrates cerca de 460-337 aC, e depois por Discórides, no primeiro século (40-80 dC) . No segundo século (130-200 dC), Galeno prescreveu a Valeriana para insônia. O médico italiano Andrea Mattiolo observou, desde o século VI, que a planta demonstrava estranhos efeitos sobre o comportamento de seres humanos e animais. No século VII, após a cura do príncipe romano Fabio Collona, descobriu possuir poder antiepiléptico.

Acredita-se que as virtudes medicinais tenham sido comentadas pela primeira vez por um médico egípcio do século IX. Em torno do ano 1000, falava-se como sendo um medicamento capaz de curar uma série de doenças, sobretudo nervosismo e epilepsia. Em meados do século XIV, foi considerada estimulante. No século XVI, foi utilizada para tratar tremores, dor de cabeça e palpitações cardíacas. Na Segunda Guerra Mundial, foi usada na Inglaterra para aliviar o estresse de ataques aéreos. Mais recentemente, o médico Monpellier Lazàre Rivière, conclui que a planta diminuía a sensibilidade nervosa e era dotada de perceptível efeito sobre o sistema nervoso central e poderia controlar a epilepsia. O efeito sedativo da Valeriana tem sido reconhecido desde o século XVIII na Europa, e desde então tem sido utilizada para distúrbios do sono (FERNANDÉZ-SAN-MARTíN et al, 2010).

Encontra-se descrita em várias edições do Dispensatório dos Estados Unidos, listada em compêndios oficiais em todo o mundo e várias espécies continuam sendo incluídas em farmacopéias de diversas nações.

A Valeriana está entre as ervas mais vendidas nos Estados Unidos. As vendas entre julho de 1997 e 1998 totalizaram \$8 milhões (BREVOORT, 1998). Em 2005, classificou-se como sendo a décima terceira erva mais vendida, com vendas estimadas de \$3,4 milhões (BLUMENTHAL; FERRIER; CAVALIERE, 2006). Em 2009, foi classificada como a décima primeira substância alimentar botânica mais vendida nos Estados Unidos (NATIONAL TOXICOLOGY PROGRAM, 2009).

Kennedy (2005, citado por NATIONAL TOXICOLOGY PROGRAM, 2009), analisou o Encontro Nacional de Saúde (National Health Interview), conduzido pelo Centro Nacional de Estatísticas da Saúde (National Center for Health Statistics), e encontrou que 5,6\% dos entrevistados relataram o uso de Valeriana nos últimos 12 meses. Stasio et al (2008, citado por NATIONAL TOXICOLOGY PROGRAM, 2009), realizaram um levantamento com 201 estudantes universitários de faculdade privadado sudeste dos Estados Unidos, 19 (9,5\%) participantes relataram ter utilizado a Valeriana 
para ansiedade na semana anterior, uma porcentagem relativamente maior que a relatada em 2002 pelo NHIS (US National Health Interviel Survey). Estes dados sugerem a ampla utilização da Valeriana.

O objetivo principal deste trabalho é avaliar a efetividade da Valeriana no tratamento da insônia, por meio de uma revisão de artigos científicos publicados.

\section{MÉTODOS}

Para a realização deste estudo, foi empregado o método de revisão sistemática da literatura, por meio da busca de artigos científicos publicados em diversas bases de dados, com destaque para o sistema MEDLINE; a Cochrane Library; BioMed Central; SciELO (Scientific Electronic Library Online); PubMed; ScienceDirect; LILACS; Portal Periódicos CAPES; MICROMEDEX. Foram realizadas buscas utilizando as seguintes palavras-chaves: "valeriana", "valerian", "Valeriana officinalis"; "insônia", "insomnia", "plantas medicinais", "medicinal plants", "distúrbios do sono", "sleep disorders". A pesquisa foi realizada entre os meses de maio a outubro de 2010.

Não houve restrição quanto à língua das publicações, já que a maioria dos artigos científicos foi publicada em inglês; e nem quanto a data das publicações, uma vez que existem relatos antigos que comprovam a eficácia da Valeriana no tratamento da insônia. Limitou-se apenas a relatórios originais e completos, excluindo os resumos, opiniões e editoriais. Também foram excluídos estudos não realizados em seres humanos e estudos que utilizaram preparações de Valeriana combinada com outras substâncias, como ervas e outros medicamentos, para não interferirem nos resultados. Foram selecionados ensaios que compararam a Valeriana com o grupo controle (placebo). Não houve restrição quanto ao emprego de participantes saudáveis ou com relatos de distúrbios do sono, e quanto a ensaio duplo-cego, randomizado ou cruzado.

\section{RESULTADOS E DISCUSSÕES}

Inicialmente foram identificados 110 artigos, destes foram eliminados 53 que não eram ensaios clínicos, 12 que eram estudos realizados em ratos, 14 que testaram Valeriana com outras ervas, 10 que testaram com medicamentos e 2 que não tiveram o sono como objetivo principal. Somente os artigos que preencheram os requisitos apresentados foram incluídos na revisão, totalizando 15 artigos e 4 revisões sistemáticas.

Um estudo clínico, randomizado, duplo-cego, cruzado, controlado com placebo, realizado por Leathwood et al (1982), comparou 400mg de extrato aquoso de raiz de Valeriana com uma preparação comercial de $60 \mathrm{mg}$ de Valeriana (Hova®) e placebo, em um total de 128 voluntários saudáveis. Cada participante testou 9 
amostras (3 placebos, 3 Valeriana e 3 Hova $\circledR$ ) em ordem aleatória de tomada uma hora antes de dormir por nove noites não consecutivas. Os efeitos foram medidos através de questionários na manhã seguinte. Foram avaliados os efeitos das doses únicas tomadas em ordem aleatória para a latência do sono, qualidade do sono, sonolência ao acordar, despertar a noite e recordação do sono. Os indivíduos que receberam extrato de Valeriana apresentaram uma melhora subjetiva estatisticamente significativa em relação ao placebo no tempo de latência do início do sono e na qualidade do sono, sendo este mais notável em pacientes com sono irregular, fumantes e pessoas com longa latência do sono. Despertar noturno, lembrança do sonho e sonolência na manhã seguinte foram relativamente pouco afetados pela Valeriana. A preparação comercial não apresentou melhora estatisticamente significativa nessas medidas. Significativo aumento na sonolência no dia seguinte foi relatado com Hova ${ }^{\circledR}$ em relação à Valeriana e placebo. Foi relatado somente um efeito adverso (náusea), mas não foi possível relatar a qual grupo pertencia. O significado clínico da utilização da Valeriana para o tratamento da insônia não pode ser determinado a partir dos resultados obtidos neste estudo, pois a insônia não era requerida para a participação do ensaio. Além disso, o estudo teve uma alta taxa de retirada de participantes $(22,9 \%)$, o que pode ter influenciado os resultados.

Leathwood e Chauffard (1982-83) realizaram um estudo cruzado, controlado por placebo, com 10 jovens saudáveis do sexo masculino (idade média 20 anos) que dormiram quatro noites no laboratório, com registros de medidas do eletroencefalograma (EEG) todas as noites. Em duas noites receberam $400 \mathrm{mg}$ de extrato aquoso de Valeriana e nas outras duas receberam placebo. A análise do questionário mostrou que a Valeriana possui um leve efeito sedativo em relação aos critérios subjetivos, ou seja, diminuiu a latência do sono, despertar noturno, e melhorou a qualidade do sono. Em relação aos resultados do eletroencefalograma (EEG), a latência do sono foi menor e aumentou a latência para o primeiro despertar, mas as medidas não alcançaram resultados estatisticamente significativos. O estudo analisou algumas abordagens críticas para testar a suposta sedação leve e sugeriu uma abordagem racional para analisar os efeitos.

Kamm-Khol, Jansen e Brockmann (1984), em um ensaio randomizado, duplocego, investigaram 80 pacientes idosos cronicamente doentes com distúrbios no sono em hospitais geriátricos. Os participantes receberam $90 \mathrm{mg}$ de extrato aquoso de Valeriana (Baldrian Dispert@) ou placebo três vezes ao dia por 14 dias. Foram utilizados dois questionários validados e uma escala de avaliação do sono. Maior proporção de pessoas com uso de Valeriana relatou que a latência do início do sono e o tempo de duração do sono melhoraram em relação aos indivíduos que fizeram uso do placebo (29 do grupo Valeriana versus 6 do grupo placebo para a latência do sono, e 26 do grupo Valeriana versus 10 do grupo placebo para a duração do sono). Entretanto, este estudo 
não incluiu as medidas objetivas do sono, somente baseou-se em questionários. O único efeito adverso relatado foi vertigem em dois pacientes de cada grupo.

Gessner e Klasser (1984), em um ensaio randomizado, cruzado, duplo-cego, controlado com placebo, conduziram um estudo com 11 jovens (idade média 26 anos) com distúrbios do sono, que dormiram três noites não consecutivas no laboratório do sono após tomar $60 \mathrm{mg}$ ou $120 \mathrm{mg}$ de extrato aquoso de Valeriana (Harmonicum Much () ) ou placebo. Foram realizadas medidas polissonográficas do sono e questionários foram completados na manhã seguinte. Com ambas as dosagens ocorreram ligeira redução do sono REM e diminuição do estágio 4 do sono (sono profundo). Ao contrário, ocorreu um pequeno aumento dos estágios do sono acordado, 1 e 2, e um aumento no estágio 3. Após a administração de 120mg de Valeriana, a freqüência da fase REM diminuiu na primeira metade da noite, enquanto que na segunda parte da noite, aumentou excessivamente. Mudanças nas medidas dos movimentos oculares, miograma e pulsação durante o sono ou intensidade beta do eletroencefalograma (EEG) durante o sono REM, mostraram um efeito hipnótico maior para a dosagem de $120 \mathrm{mg}$, sugerindo um efeito dose-dependente, com efeito máximo ocorrendo entre 2 e 3 horas após a administração. Os voluntários não relataram mudanças subjetivas no tempo ou duração do sono, efeitos de ressaca ou efeitos adversos.

Outro estudo duplo-cego, randomizado, cruzado, controlado com placebo, realizado por Leathwood e Chauffard (1985), investigou 8 pacientes (idade média 45 anos) com insônia leve tratados com 450mg ou 900mg de extrato aquoso da raiz de Valeriana ou placebo por quatro noites não consecutivas. Um medidor da ativação da pulsação registrou movimentos durante a noite e os voluntários responderam questionários após acordar. Utilizando o primeiro período de 5 minutos consecutivos sem movimentos como um critério de início do sono, houve uma diminuição significativa na latência do sono com dose única de $450 \mathrm{mg}$ e $900 \mathrm{mg}$ de Valeriana, sendo o sono mais estável durante o primeiro quarto da noite e sem efeito no tempo total do sono em comparação com o placebo. A maior dose de Valeriana não produziu melhorias na latência do sono, mas causou maior sonolência ao despertar em relação ao placebo. Não houve relato de efeitos adversos neste estudo.

Ainda neste mesmo ano, um pequeno estudo duplo-cego, cruzado, controlado com placebo, realizado por Balderer e Borbély (1985), avaliou o efeito do extrato aquoso de raiz Valeriana (450mg e 900mg) comparado com placebo em 18 voluntários saudáveis. Um grupo de 10 pessoas ( 5 homens e 5 mulheres com idade média de 32 anos) participaram do estudo em casa e outro grupo de 8 pessoas (4 homens e 4 mulheres com idade média de 22 anos) permaneceram no laboratório. O grupo que permaneceu em casa, recebeu 4 cápsulas (2 doses de Valeriana e placebo) administradas 30 minutos antes de deitar na quarta ou quinta-feira por três semanas 
consecutivas (uma semana transcorrida entre as noite experimentais). No grupo do laboratório, após a primeira noite de adaptação, os participantes administraram quatro cápsulas 30 minutos antes de deitar nas quatro próximas noites; em uma das noites as cápsulas continham $900 \mathrm{mg}$ de Valeriana e nas outras noites placebo. Os resultados foram avaliados através de questionários e escalas de auto-avaliação e atividade motora noturna. No grupo do laboratório também foram realizadas medida objetiva polissonográfica do sono e análise do espectro do eletroencefalograma (EEG). Nas condições em casa, ambas as doses de Valeriana reduziram a latência do sono e o tempo de vigília após o início do sono em relação ao placebo, mas não houve diferença significativa entre as doses. O número de despertares noturno também diminuiu, mas não foi significativo. A auto-avaliação da qualidade do sono não apresentou diferenças entre os tratamentos. O tempo da atividade motora noturna foi maior na terceira metade da noite e reduziu no último terço da noite, a qual sugere um efeito dose-dependente. No terço médio da noite, a dose mais elevada induziu maior atividade noturna em relação ao placebo. Não houve evidência de efeito no terço inicial da noite. Já no grupo do laboratório, no qual somente a maior dose foi testada, não houve diferença significativa entre os resultados de Valeriana e placebo. Para medidas objetivas e subjetivas, a latência do sono e o tempo de despertar após o início do sono apresentaram valores menores com a administração de Valeriana. No grupo de Valeriana, a atividade motora noturna na segunda noite foi maior e na terceira noite foi menor. A redução da atividade da segunda para a terceira noite ocorreu somente com a administração de Valeriana. Além disso, não houve diferença nas fases do sono. Também não foi observado diferença nos espectros do eletroencefalograma (EEG) de Valeriana e placebo. Os autores sugeriram que o extrato aquoso de Valeriana exerce leve ação hipnótica, entretanto vários aspectos farmacológicos (farmacocinética e incidência de efeitos colaterais) necessitam ser esclarecidos antes da aplicação da Valeriana na farmacoterapia da insônia.

Schulz, Stolz e Müller (1994), em um estudo randomizado, controlado com placebo, investigaram $405 \mathrm{mg}$ de extrato aquoso de Valeriana (Valdispert@) ou placebo em 14 idosos do sexo feminino, idade média de 62 anos, com dificuldade no início e manutenção do sono (6 indivíduos receberam placebo e 8 indivíduos receberam Valeriana) por um período de 7 dias. O sono foi avaliado subjetivamente através de questionários validados ao longo de sete dias e objetivamente através da polissonografia em três noites no laboratório, com intervalos de uma semana. O grupo de Valeriana apresentou um aumento significativo nas ondas lentas do sono (SWS) e diminuição no estágio 1 do sono. Não foi observado efeito no tempo do início do sono ou tempo de vigília após o início do sono. O sono REM manteve-se inalterado. Também não houve efeito na qualidade do sono observada pela auto-avaliação. Este estudo foi limitado pelo número pequeno de pacientes. 
Vorbach, Gortelmeyer e Bruning (1996) demonstraram o efeito da Valeriana em um estudo randomizado, duplo-cego, controlado com placebo utilizando 121 pacientes (idade média 47 anos) com insônia não orgânica, não sofrendo de depressão e sem a possibilidade do uso de medicação que possa interferir como o sono. Foram administrados $600 \mathrm{mg}$ de extrato etanólico de Valeriana (LI 156) ou placebo por 28 dias consecutivos. A eficácia foi avaliada através de quatro escalas validadas. A Valeriana apresentou resultado significativamente melhor que o placebo na escala clínica de impressão global (CGI) após 14 dias e em duas outras medidas, após 28 dias. Houve relato de melhora no sono em $66 \%$ do grupo que recebeu Valeriana em comparação com $26 \%$ do grupo que recebeu placebo. Somente dois pacientes de cada grupo relataram efeitos adversos; e os efeitos associados à Valeriana foram dor de cabeça e sensação de desorientação pela manhã, que podem ser interpretados como efeitos de ressaca.

Um estudo randomizado, duplo-cego, controlado com placebo, cruzado, realizado por Donath et al (2000), avaliou a curto prazo (dose única) e a longo prazo (14 dias com doses múltiplas) os efeitos de $600 \mathrm{mg}$ do extrato etanólico de Valeriana em parâmetros objetivos e subjetivos do sono. O estudo envolveu 16 pacientes (4 do sexo masculino e 12 do sexo feminino) com insônia previamente confirmada pela polissonografia, sem doenças agudas e com idade média de 49 anos. Foram analisados oito registros polissonográficos: dois registros (basal e a noite) em cada momento em que os efeitos da Valeriana e do placebo a curto e a longo prazo foram testados. A eficácia do sono foi o alvo variável do estudo. Foram analisados os parâmetros objetivos do sono, avaliando o estágio do sono e o índice de excitação. Os parâmetros subjetivos, como a qualidade do sono, sensação pela manhã, desempenho durante o dia, duração da latência do sono, tempo e período do sono foram avaliados através de questionários. A dose única de Valeriana não demonstrou efeito na estrutura do sono e na avaliação subjetiva do sono. Após o tratamento com doses múltiplas, foi observado aumento significativo da polissonografia basal tanto para a Valeriana quanto para o placebo. A latência do sono de ondas lentas (SWS) reduziu com a administração de Valeriana em comparação com o placebo. O percentual do tempo de ondas lentas do sono na cama (TIB) aumentou após tratamento a longo prazo com a preparação de Valeriana, e ocorreu uma redução na latência subjetiva e objetiva do sono. Também foi relatado melhora na estrutura do sono como o aumento na porcentagem REM e redução no percentual NREM1 com placebo e Valeriana. Com doses múltiplas de Valeriana administradas por 14 dias, foram relatados apenas 3 eventos adversos (enxaqueca e efeitos gastrointestinais) em pacientes tratados com Valeriana em comparação com 18 eventos em pacientes que receberam placebo. Os autores concluíram que o tratamento com Valeriana mostrou efeitos positivos na estrutura do sono e percepção do sono em pacientes com insônia, podendo ser recomendado para 
o tratamento de pacientes com insônia psicofisiológica leve.

Francis e Dempster (2002) realizaram um estudo randomizado, duplo-cego, controlado com placebo para avaliar o potencial da Valeriana $(20 \mathrm{mg} / \mathrm{kg}$ de peso de V.edulis) no tratamento de distúrbios do sono em crianças com um déficit intelectual (ID). Foram utilizadas 5 crianças com diferentes déficits intelectuais e diferentes problemas do sono primário e foram submetidas a 8 semanas de acompanhamento. Com relação ao valor basal e ao placebo, o tratamento com Valeriana reduziu significativamente a latência de sono e o tempo de vigília noturna, prolongou o tempo total de sono e melhorou a qualidade do sono. O tratamento foi aparentemente mais eficaz em crianças com déficits do que com hiperatividade. Os autores concluíram que embora os resultados sejam preliminares e necessitam de replicação, não há evidências que sugerem que a Valeriana pode ser útil no tratamento seguro e eficaz a longo prazo nas dificuldades para dormir em crianças com ID's, necessitando, portanto, de uma investigação mais aprofundada.

Coxeter et al (2003) realizaram um estudo para evidenciar a eficácia da Valeriana no tratamento da insônia crônica em Queensland, na Austrália. Foram avaliados 42 pacientes (idade média 54 anos), dos quais apenas 24 (57\%) continham os requisitos exigidos para a sua inclusão no ensaio. Os participantes administraram $450 \mathrm{mg}$ de extrato de raiz de Valeriana por 21 dias. A resposta da Valeriana foi boa para 23 participantes (96\%) que avaliaram o seu nível de energia no dia anterior, e ruim para os 24 participantes (100\%) que responderam sobre o tempo total do sono e para 23 participantes (96\%) que responderam sobre o número de despertares noturno e "alívio pela manhã". A proporção de sucessos no tratamento variou de 0,35 para 0,55 para as seis variáveis do resultado do sono (latência do sono, número de despertares noturno, tempo total do sono, qualidade do sono, nível de percepção de "alívio" pós-repouso, nível de energia no dia anterior). O estudo mostrou uma tendência positiva para a Valeriana, mas não foi significativamente superior em relação ao placebo. Não houve diferença significativa no número, distribuição ou gravidade de efeitos colaterais entre Valeriana e placebo. Os autores concluíram que a Valeriana não apresentou ser muito melhor do que o placebo na promoção do sono ou fatores relacionados ao sono para cada paciente ou para todos os pacientes como um grupo.

Diaper e Hindmarch (2004), em um estudo duplo-cego, randomizado, cruzado, compararam 300mg e 600mg de extrato etanólico de Valeriana (LI 156 Sedonium $®$ ) com placebo em 16 indivíduos ( 5 do sexo masculino e 11 do sexo feminino, idade média 56 anos) com sono leve. Os participantes dormiram em um laboratório do sono, após uma dose de $300 \mathrm{mg}$ de Valeriana, $600 \mathrm{mg}$ de Valeriana ou placebo. Foram realizadas medidas do eletroencefalograma (EEG) do sono e testes psicométricos padronizados para avaliar a eficácia da Valeriana. Não foram encontradas diferenças significativas nos resultados polissonográficos ou subjetivos do sono (eficiência do sono, latência do 
sono, vigília, estágios do sono e avaliação da qualidade do sono). Os autores concluíram que a Valeriana nas doses utilizadas é ineficaz para distúrbios do sono. Portanto, são necessárias mais pesquisas em relação às doses terapêuticas, tipos de preparação e tempo de tratamento ideal para se obter um efeito terapêutico.

Oxman et al (2007) selecionaram os participantes através um programa de saúde semanal da televisão da Noruega. O estudo randomizado, duplo-cego, controlado com placebo selecionou 405 participantes com idade entre 18 e 75 anos e apresentando sintomas de insônia. Os voluntários ficaram duas semanas sem tratamento e receberam aleatoriamente Valeriana ou placebo durante 2 semanas. $O$ primeiro resultado obtido foi uma pequena melhora na qualidade do sono auto-relatada pelos participantes, mas a diferença entre o grupo de Valeriana e o grupo placebo não foi estatisticamente significativa. No final do tratamento, em relação à questão global de auto-avaliação, um maior número de participantes do grupo Valeriana relatou que conseguiram uma melhora no sono. Foi relatada uma tendência de melhora no grupo de Valeriana para despertares noturno e duração do sono. Não foram relatados efeitos adversos graves e não houve diferença significativa em efeitos adversos agudos. Os autores concluíram que a Valeriana parece ser segura, mas tem poucos efeitos benéficos, principalmente na insônia, em comparação com o placebo.

Um estudo randomizado, duplo-cego, cruzado, controlado com placebo realizado por Taibi et al (2009), avaliou a eficácia da Valeriana na insônia. No experimento foram utilizadas 16 mulheres idosas (idade média 69 anos) com insônia, que administraram $300 \mathrm{mg}$ de extrato de Valeriana ou placebo 30 minutos antes de dormir por duas semanas. O sono foi avaliado no laboratório por auto-relato e polissonografia basal, e também no início e no fim de cada fase do tratamento (9 noites no laboratório), e em casa por registros diários do sono e actigrafias. Não teve diferença significativa entre Valeriana e placebo após administração de dose única ou após duas semanas de administração noturna em relação a latência do sono, despertar após o início do sono, eficácia do sono e qualidade do sono. Após duas semanas de administração noturna de Valeriana, ocorreu um aumento significativo no tempo de despertar após o início do sono em comparação com o placebo. Os efeitos colaterais foram menores e não foram diferentes entre Valeriana e placebo. Neste estudo os autores concluíram que a Valeriana não apresentou melhora no sono em mulheres idosas com insônia.

Herrera-Arellano et al (2001) realizaram um ensaio duplo-cego, cruzado, controlado com placebo para avaliar o efeito hipnótico e a segurança de $250 \mathrm{mg}$ do extrato alcoólico padronizado de Valeriana edulis no tratamento da insônia em 20 voluntários. O extrato de Valeriana officinalis foi utilizado nas mesmas dosagens como controle positivo. No laboratório do sono, a polissonografia foi realizada para avaliar a quantidade e a arquitetura do sono, sonolência pela manhã, memória e efeitos 
colaterais. Os experimentos foram realizados por quatro noites consecutivas (8 horas cada). De acordo com resultados polissonográficos, $V$. edulis reduziu os episódios de despertar noturno, enquanto que ambos os tratamentos aumentaram o movimento rápido dos olhos (sono REM), este significativamente melhorado pela $V$. officinalis. Outros resultados polissonográficos não tiveram diferença estatística. Os extratos de Valeriana diminuíram o tempo das fases 1 e 2 do sono não-REM e aumentaram o sono delta, produzindo assim efeitos benéficos na arquitetura do sono. Testes clínicos validados demonstraram que ambas as espécies reduziram a sonolência pela manhã, melhorada por $V$. officinalis, e não afetaram a memória aterógrada. Foram observados apenas três casos de efeitos colaterais leves. Os resultados confirmaram o efeito hipnótico e a segurança no tratamento agudo da $V$. edulis e $V$. officinalis em pacientes cominsônia.

Algumas revisões sistemáticas sobre a eficácia da Valeriana sobre a insônia foram realizadas, mas chegam a diferentes conclusões. Uma revisão sistemática de ensaios clínicos descrita por Stevinson e Ernst (2000), analisou os efeitos da Valeriana em pacientes com insônia. Foram utilizados somente ensaios clínicos randomizados, duplo-cegos, controlados com placebo, de mono-preparações de Valeriana e estudos realizados em humanos. Nove estudos preencheram os critérios de inclusão (três examinaram o efeito cumulativo da administração em dias consecutivos e seis mediram as respostas agudas com doses individuais). Os autores sugeriram que há tendência de ensaios com resultados negativos não terem sido publicados e então não serem incluídos na revisão. Nenhum ensaio obteve sucesso no controle da condução da "cegueira". Alguns estudos foram rigorosos, mas nenhum foi completamente sem falha. Diversos ensaios utilizaram açúcar como placebo, o que diferencia muito o sabor de cápsulas de Valeriana, podendo alterar os resultados. Apenas três ensaios descreveram seus processos de randomização. Não houve controle quanto à prática de exercícios durante o tratamento, consumo de alimentos, álcool ou cafeína, os quais podem interferir no sono. Vários estudos utilizaram indivíduos saudáveis. Dos ensaios que utilizaram voluntários com distúrbios no sono, somente dois fizeram uso de critérios rigorosos de inclusão. Como a insônia é mais prevalente com o aumento da idade e em mulheres, os autores sugeriram que a realização de estudos em jovens do sexo masculino e estudos em idosos do sexo feminino poderiam ajudar a encontrar resultados diferentes.

A metodologia utilizada nos ensaios não era muito potente e o tamanho das amostras em alguns estudos era pequeno, com seis estudos tendo menos que 15 participantes. O resultado do sono no laboratório pode ter sido melhor devido ao fato de o paciente poder fugir da rotina normal na qual a insônia pode ser uma resposta condicionada. Os autores relatam que não existe confiança da polissonografia em relação à medida da qualidade do sono e também é um método invasivo, o que 
restringe sua utilização. Por isso, três estudos utilizaram medidores de pulsação, mas não existem provas de que este método seja confiável para a medida da latência do sono. As doses utilizadas nos ensaios foram diferentes. De seis estudos que avaliaram o efeito de doses únicas de Valeriana, somente três relataram resultados positivos comparados com placebo. Dois estudos apresentaram melhora nos parâmetros relacionados com o sono em pacientes com insônia que receberam administração repetida ao longo de duas a quatro semanas, mas não apresentaram diferenças entre eles. Relatos de efeitos adversos com a administração de Valeriana foram escassos, e os que foram notificados foram leves e semelhantes ao placebo. Os autores concluíram que as evidências não foram suficientes para justificar o uso da Valeriana no tratamento a curto prazo da insônia. Alguns estudos sugeriram que a Valeriana pode ter efeitos agudos e cumulativos sobre o sono, mas nem todos produziram resultados positivos. Os autores sugerem que os testes devem ser mais rigorosos, as amostras devem ser maiores, deve-se ter controle de variáveis e utilizar medidas de resultados validadas. Os ensaios devem examinar efeitos agudos e repetidos e comparar diferentes doses. E, também, deve-se dar maior atenção aos efeitos adversos e efeitos de ressaca.

Bent et al (2006) realizaram uma revisão sistemática e meta-análise de 16 estudos randomizados, controlados com placebo, com um total de 1093 participantes. A maioria dos estudos incluiu pacientes saudáveis. Em geral, o tamanho da amostra foi pequeno, com oito estudos examinando menos que 25 pacientes, e a maioria com problemas metodológicos significantes. Além do baixo índice de qualidade média do estudo, que mede os problemas relacionados com comportamento ou descrição da randomização, "cegueira", e retirada dos participantes, existiram outros inúmeros problemas que limitaram a capacidade de extrair conclusões sobre a eficácia e segurança da Valeriana. Houve variação nas preparações e dosagens de Valeriana, duração do tratamento e avaliação dos resultados. De sete estudos que usaram uma escala visual analógica para avaliar a mudança na qualidade do sono, somente dois estudos observaram melhoria no grupo de Valeriana, mas não apresentaram informações para determinar se essas mudanças foram comparadas com o placebo. Os autores relataram que há evidências de que ocorreu influência de publicação, o que sugere que estudos com resultados negativos podem não ter sido publicados na revisão, levando a uma super-estimação do efeito da Valeriana. De nove ensaios que avaliaram a eficácia da Valeriana na latência subjetiva do início do sono, apenas quatro apresentaram aumento estatisticamente significativo da latência em minutos, apresentando pelo menos uma tendência favorecendo a Valeriana. Apenas dois estudos determinaram que a Valeriana foi padronizada para o ácido valerênico, que acredita ser um dos componentes ativos da erva. De seis estudos que relataram medida de ressaca pela manhã, nenhum obteve diferença entre Valeriana e placebo. Não houve alterações significativas em relação à eficácia do sono, duração do sono, 
tempo de cada estágio sono, medida de latência do início do sono, movimento rápidos dos olhos na latência do início do sono e número de despertares noturno. Apenas um ensaio clínico identificou ocorrência de efeitos adversos no grupo de Valeriana (diarréia). A maioria dos estudos não descreveu a análise dos efeitos adversos, como recomendado recentemente. Os autores concluíram que a Valeriana pode melhorar a qualidade do sono, mas devido a problemas metodológicos dos estudos incluídos, a capacidade de tirar conclusões definitivas é limitada. Eles recomendam que sejam realizados mais estudos com maior rigor metodológico, avaliando produtos de Valeriana padronizados para níveis específicos de ingredientes ativos suspeitos e que centralizem na detecção de efeitos adversos, incluindo tolerância e efeitos de retirada.

A revisão sistemática de Taibi et al (2007) utilizou 37 ensaios clínicos, com um total de 1900 pacientes. Utilizaram vários tipos de produtos (espécies, métodos de extração e combinação de ervas diferentes) e as características das amostras estudadas foram diferentes (indivíduos saudáveis e indivíduos com insônia). Dosagens e duração do tratamento não foram enfatizadas. Não excluíram outros tipos de distúrbios do sono. Não conseguiram realizar uma meta-análise devido à qualidade variável das pesquisas. Os autores sugerem que os dados sobre a Valeriana em grupos paralelos e cruzados não deverão ser combinados na mesma meta-análise, pois aumenta o risco de erros. Poucos estudos controlados com placebo de duas semanas de tratamento ou mais relataram melhora significativa com Valeriana. Relataram que os estudos realizados com extrato etanólico eram recentes e de alta qualidade. Ensaios com extrato aquoso foram realizados entre 1970 e 1980 e com qualidade mista. No estudo de maior qualidade (com extrato etanólico) a Valeriana não teve efeito significativo superior ao placebo na insônia. Os resultados não foram eficazes na melhoria subjetiva do sono. Algumas evidências sugeriram que a Valeriana pode melhorar os resultados no sono, mas o efeito do placebo de muitos ensaios não pode ser excluído. Os resultados objetivos não apóiam a eficácia da Valeriana para melhorar o sono. Foram relatados efeitos colaterais leves como dor de cabeça, tontura, sonolência, náusea e diarréia. Não foram relatados sintomas hepáticos ou alteração nas funções hepáticas. Foi demonstrado que o dano ao DNA em células epiteliais humanas ocorre somente com doses elevadas. Há poucas evidências que comprovem a interação com medicamentos. Alguns estudos indicaram que não deve ser associado Valeriana com outros sedativos, como antihistamínicos e álcool. Os autores concluíram que a Valeriana é uma planta aparentemente segura, com poucos efeitos colaterais, mas as evidências não comprovam sua eficácia na redução de distúrbios do sono ou sintomas da insônia.

Fernández-San-Martín et al (2010) realizaram uma meta-análise de 18 ensaios clínicos randomizados, controlados com placebo. A qualidade do sono foi avaliada através da variável dicotômica (sim ou não), escalas visuais analógicas, e também o 
tempo de latência do início do sono em minutos através da medida polissonográfica ou de questionários de auto-avaliação. $O$ tamanho da amostra foi pequeno na maioria dos estudos, entre 5 e 434 pacientes. Houve variação na dosagem, tempo de tratamento e tipo de Valeriana utilizado. Foram utilizados voluntários saudáveis somente em três estudos. As condições de execução foram variadas (no laboratório, em casa, sem restrição quanto ao consumo de outras substâncias). Dez estudos avaliaram o efeito da Valeriana na latência do sono em comparação com o placebo, mas não produziram resultados significativos. Somente seis ensaios avaliaram a qualidade do sono comparando Valeriana com placebo, destes apenas dois utilizaram questionários validados, o restante obteve questionários projetados pelos próprios autores. Nos grupos com maior rigor metodológico, o efeito da Valeriana na qualidade do sono não foi significativo. Nesta revisão a Valeriana melhorou a qualidade do sono em relação à variável dicotômica. Com a escala visual analógica, não obteve melhora no tempo de latência em minutos para iniciar o sono ou melhora na qualidade do sono. Os ensaios incluídos foram heterogêneos e não cumpriram com os critérios de qualidade. Uma quantidade elevada de estudos (40\%) foi pouco rigorosa. Houve falta de padronização na medida da qualidade do sono, falta de apresentação de dados e limitação na avaliação dos efeitos adversos. A análise realizada com variáveis quantificáveis (latência do sono em minutos, qualidade do sono medida com VAS ou escalas quantificáveis), não relatou significativa melhora entre Valeriana e placebo. As medidas da latência do sono e qualidade do sono em escalas analógicas visuais não foram significativas. A ausência de aroma no placebo pode comprometer os resultados em favor da Valeriana. Pacientes tratados com Valeriana subjetivamente relataram aumento na qualidade do sono, mas isso não ocorreu com medidas quantitativas. Os resultados mostraram melhora significativa na insônia tanto com Valeriana quanto com placebo. Os autores relatam que nesta revisão existiram várias limitações. A elegibilidade dos resultados foi restrita. Não houve influência de publicação e nem de idioma. Mais estudos foram incluídos nesta meta-análise e foi limitada para pacientes que usaram Valeriana sem a combinação de outras ervas. Foram incluídas duas metaanálises com resultados inconclusivos. Os autores relataram que através dos resultados dicotômicos, a Valeriana pode ser eficaz para a melhora subjetiva da insônia, embora a eficácia não foi demonstrada com medidas quantitativas e objetivas. Foram relatados dois efeitos adversos leves (desordens gastrointestinais como diarréia, epigastralgia, náusea; e efeitos no sistema nervoso central como dor de cabeça, nervosismo e sonolência), contudo não foram diferentes com o placebo, com exceção da diarréia. Os ensaios clínicos mais recentes de alta qualidade metodológica e tamanhos de amostras suficientes, não foram conclusivos. Os autores concluíram que os resultados dos estudos utilizados não foram promissores no uso da Valeriana para a insônia e recomendam futuras investigações. 
Com base na análise dos resultados obtidos nos estudos incluídos na revisão, alguns ensaios sugerem um efeito promissor da Valeriana no tratamento da insônia, enquanto que a maioria dos ensaios relata que há a necessidade de mais estudos para chegar a uma conclusão definitiva do efeito da Valeriana.

A dificuldade em avaliar a eficácia da Valeriana no tratamento da insônia devese ao fato de que houve uma variação metodológica utilizada nos diferentes ensaios. As doses e os esquemas de dosagens foram diferentes em diversos estudos. O tempo de tratamento utilizado variou muito entre os ensaios. Foram utilizadas diferentes preparações de Valeriana sem dar atenção especial ao tipo e origem das espécies e a técnica utilizada na extração (com etanol e água, metanol e água ou apenas água), uma vez que os componentes químicos variam de acordo com a espécie e com o método de extração utilizado, o que pode influenciar nas comparações dos resultados para obter uma conclusão precisa. Os resultados foram avaliados de maneira diferente entre os ensaios clínicos. Os métodos utilizados não foram rigorosos e alguns utilizaram medidas não validadas, como questionários produzidos pelo próprio autor e medidas de pulsação e polissonografia, que não existem provas de que são medidas confiáveis. Alguns estudos não foram duplo-cegos e nem randomizados, o que poderia influenciar nos resultados. Não houve restrição quanto ao consumo de alimentos, álcool, cafeína e medicamentos que possam interferir no sono. Os efeitos adversos não foram analisados em todos os ensaios. Alguns relataram que podem ter ocorrido resultados negativos que não foram publicados, portanto não foram incluídos nos estudos. Não houve restrição em utilizar indivíduos saudáveis ou com distúrbios do sono e nem com relação à idade e o sexo, o que poderia produzir resultados diferentes. Além disso, a quantidade de voluntários utilizada nos ensaios foi relativamente pequena. Não foi considerada a alta taxa de retirada dos participantes dos estudos. Também não foi analisada a quantidade dos constituintes químicos existentes em cada preparação, para avaliar qual o componente ativo poderia ser responsável pelo efeito da Valeriana.

De acordo com alguns estudos, não há um consenso científico quanto aos componentes ativos da Valeriana, e sua atividade pode resultar da ação de um único composto ou da interação entre compostos múltiplos. A influência da extração sobre o efeito fisiológico da Valeriana permanece desconhecida, pois ainda não foi determinado qual é o componente responsável pelo efeito ou se existe uma ação sinérgica de vários compostos. Duas categorias de constituintes têm sido propostas como a principal fonte do efeito sedativo da Valeriana (NATIONAL INSTITUTES OF HEALTH, 2008). A primeira compreende os constituintes majoritários do óleo volátil, incluindo o ácido valerênico, que tem demonstrado propriedades sedativas em animais; entretanto, extratos de Valeriana com pouco ácido valerênico também possui propriedade sedativa, o que torna provável que outros elementos são responsáveis ou contribuem para esses efeitos. A segunda categoria compreende os iridóides, incluindo os 
valpotriatos, que em estudos in vivo demonstraram possuir propriedades sedativas; mas são instáveis e se degradam durante o armazenamento ou em ambiente aquoso, sendo improvável estarem presentes na preparação final. Embora acredita-se que os sesquiterpenos do óleo volátil sejam responsáveis pela maior parte dos efeitos da Valeriana, é provável que todos os componentes ativos de maneira sinérgica produzam uma resposta clínica (HADLEY; PETRY, 2003). Ensaios relatam que um possível mecanismo pelo qual o extrato de Valeriana pode causar sedação é aumentar a quantidade de ácido gama-aminobutírico (GABA, um neurotransmissor inibitório) na fenda sináptica (NATIONAL INSTITUTES OF HEALTH, 2008).

Valeriana é uma opção segura e tem boa tolerabilidade. Está na lista do FDA (Food and Drug Administration), sendo reconhecida como uma substância segura para consumo (GRAS - Generally recognized as safe). A maioria dos estudos sugere que a Valeriana é mais eficaz quando usada de forma contínua, ao longo de várias semanas, diminuindo a latência do sono e melhorando a qualidade subjetiva do sono. Também pode ser utilizada na descontinuação da administração dos benzodiazepínicos em pacientes com insônia. De acordo com monografias da "Comissão E", não existem contra-indicações para o seu uso (BLUMENTHAL, 1998). Poucos efeitos adversos atribuíveis à Valeriana foram relatados, entre eles dor de cabeça, tontura, prurido e distúrbios gastrointestinais, mas os efeitos similares também foram relatados com o placebo. Não existem provas da potencialização do efeito da Valeriana pela ingestão concomitante com álcool em animais e humanos, mas a associação deve ser evitada (ALBRECHT et al, 1995). Conforme estudos realizados, a Valeriana pode potencializar os efeitos dos sedativos dos barbitúricos, anestésicos e outros depressores do sistema nervoso central (ANG-LEE; MOSS; YUAN, 2001).

\section{CONCLUSÃO}

Apesar de existirem vários ensaios clínicos avaliando o efeito da Valeriana no tratamento da insônia, sua eficácia e efeitos colaterais ainda não estão totalmente estabelecidos.

A maioria dos ensaios clínicos analisados apresentou resultados promissores, mas foram inconclusivos. Dos 15 ensaios, somente 4 mostraram resultados positivos. O número de participantes nos estudos foi relativamente pequeno, com apenas 3 ensaios realizados em mais de 100 indivíduos. Somente 9 estudos selecionaram os participantes com distúrbios do sono, o que poderia interferir nos resultados. As dosagens e o tempo de tratamento utilizados foram diferentes. Os métodos utilizados para avaliar a eficácia da Valeriana foram diversos entre os ensaios, impossibilitando fazer comparações entre eles. Alguns estudos obtiveram os resultados a partir de métodos não validados. Os efeitos adversos não foram analisados em todos os 
ensaios.

Devido aos problemas metodológicos e resultados conflitantes apresentados nos ensaios clínicos, as evidências de eficácia disponíveis são consideradas fracas para justificar o uso da Valeriana no tratamento da insônia, indicando a necessidade da realização de novos estudos.

\section{REFERÊNCIAS}

ALBRECHT, M.; BERGER, W.; LAUX, P.; SCHMIDT, U.; MARTIN, C. Psychopharmaceuticals and safety in traffic. Zeitschrift fur Allegmeinmedizin. Stuttgart, v. 71, p. 1215-1225, 1995.

AMERICAN PSYCHIATRIC ASSOCIATION. Diagnostic and statistical manual of mental disorders. 4. ed (DSM-IV). Washington, DC: American Psychiatric Association, 1994.

ANG-LEE, M. K.; MOSS, J.; YUAN, C. S. Herbal medicines and perioperative care. Journal of the American Medical Association. Chicago, v. 286, n. 2, p. 208-216, 11 jul. 2001.

BALDERER, G.; BORBÉLY, A. A. Effect of valerian on human sleep. Psychopharmacology. Berlin, v. 87, n. 4, p. 406-409, 1985.

BASTIEN, C. H.; VALLIÈRES, A.; MORIN, C. M. Precipitating factors of insomnia. Behavioral Sleep Medicine. Philadelphia, v. 2, n. 1, p. 50-62, 2004.

BENT, S.; PADULA, A.; MOORE, D.; PATTERSON, M.; MEHLING, W. Valerian for sleep: A systematic review and meta-analysis. American Journal of Medicine. New York, v. 119, n. 12, p. 1005-1012, dez. 2006.

BLUMENTHAL, M. The complete German Commission E monographs: therapeutic guide to herbal medicines. Austin: American Botanical Council, p. 226-227, 1998.

BLUMENTHAL, M.; FERRIER, G. K. L.; CAVALIERE, C. Total sales of herbal supplements in United States show steady growth. HerbalGram. Austin, v. 71, p. 64-66, 2006.

BREVOORT, P. The booming U.S. botanical market: a new overview. HerbalGram. Austin, v. 44, p. 33-46, 1998. 
COXETER, P. D.; SCHLUTER, P. J.; EASTWOOD, H. L.; NIKLES, C. J.; GLASZIOU, P. $P$. Valerian does not appear to reduce symptoms for patients with chronic insomnia in general practice using a series of randomised n-of-1 trials. Complementary Therapies in Medicine. Edinburgh, v. 11, n. 4, p. 215-222, dez. 2003.

DIAPER, A.; HINDMARCH, I. A double-blind, placebo-controlled investigation of the effects of two doses of a valerian preparation on the sleep, cognitive and psychomotor function of sleep-disturbed older adults. Phytotheraphy Research. London, v. 18, n. 10 , p. 831-836, out. 2004.

DONATH, F.; QUISPE, S.; DIEFENBACH, K.; MAURER, A.; FIETZE, I.; ROOTS, I. Critical evaluation of the effect of valerian extract on sleep structure and sleep quality. Pharmacopsychiatry. Stuttgart, v. 33, n. 2, p. 47-53, mar. 2000.

FERNÁNDEZ-SAN-MARTÍN, M. I.; MASA-FONT, R.; PALACIOS-SOLER, L.; SANCHO-GÓMEZ, P.; CALBÓ-CALDENTEY, C.; FLORES-MATEO, G. Effectivenes of Valerian on insomnia: A meta-analysis of randomized placebo-controlled trials. Sleep Medicine. Amsterdam, v. 11, n. 6, p. 505-511, jun. 2010.

FRANCIS, A. J.; DEMPSTER, R. J. Effect of valerian, Valeriana edulis, on sleep difficulties in children with intellectual deficits: randomised trial. Phytomedicine. Stuttgart, v. 9, n. 4, p. 273-279, maio 2002.

GESSNER, B.; KLASSER, M. Untersuchung der Wirkung von Harmonicum Much® auf den Schlaf mit Hilfe polygraphischer EEG-Aufzeichnungen. EEG EMG Z Elektroenzephalogr Elektromyogr Verwandte Geb. Stuttgart, v. 15, n. 1, p. 45-51, mar. 1984.

HADLEY, S.; PETRY, J. J. Valerian. American Family Physician. Kansas City, v. 67, n. 8, p. 1755-1758, 15 abr. 2003.

HAJAK, G.; SINE Study Group. Epidemiology of severe insomnia and its consequences in Germany. European Archives of Psychiatry and Clinical Neurosciences. Berlin, v. 251, n. 2, p. 49-56, 2001.

HERBALIST, R. U. American Herbal Pharmacopoeia and Therapeutic Compendium: Valerian root (Valeriana officinalis): analytical quality control, and therapeutic monograph. Santa Cruz, abr. 1999. Disponível em: <http://www.herbalahp.org/documents/sample/valerian.pdf>. Acesso em: 23 maio 2010. 
HERRERA-ARELLANO, A.; LUNA-VILLEGAS, G.; CUEVAS-URIOSTEGUI, M. L.; ALVAREZ, L.; VARGAS-PINEDA, G.; ZAMILPA-ALVAREZ, A.; TORTORIELLO, J. Polysomnographic evaluation of the hypnotic effect of Valeriana edulis standardized extract in patients suffering from insomnia. Planta Medica. Stuttgart, v. 67, n. 8, p. 695-699, nov. 2001.

KAMM-KOHL, A. V.; JANSEN, W.; BROCKMANN, P. Moderne baldriantherapie gegen nervöse Störungen im Senium. Medizinische Welt. Stuttgart, v. 35, p. 1450-1454, 1984.

LEATHWOOD, P. D.; CHAUFFARD, F.; HECK, E.; MUNOZ-BOX, R. Aqueous extract of valerian root (Valeriana officinalis L.) improves sleep quality in man. Pharmacology, Biochemistry and Behavior. Phoenix, v. 17, n. 1, p. 65-71, jul. 1982.

LEATHWOOD, P. D.; CHAUFFARD, F. Quantifying the effects of mild sedatives. Journal of Psychiatric Research. Oxford, v. 17, n. 2, p. 115-122, 1982-83.

LEATHWOOD, P. D.; CHAUFFARD, F. Aqueous extract of valerian reduces latency to fall asleep in man. Planta Medica. Stuttgart, v.51, p. 144-148, abr. 1985.

LEGER, D.; LEVY, E.; PAILLARD, M. The direct costs of insomnia in France. Sleep. New York, 22 supl. 2, s. 394-401, 1 maio 1999.

NATIONAL INSTITUTES OF HEALTH: Office of Dietary Supplements. Dietary supplement fact sheet: Valerian. Bethesda, 2008. Disponível em: <http://ods.od.nih.gov/factsheets/valerian.asp>. Acesso em: 23 maio 2010.

NATIONAL TOXICOLOGY PROGRAM (NTP) Board of Scientific Counselors Meeting. DEVITO, M. NTP Research Concept: Valerian (Valeriana officinalis). Department of Health and Human Services, 9-10 dez. 2009. Disponível em: $<$ http://ntp.niehs.nih.gov/NTP/About_NTP/BSC/2009/December/Draft_ResConcept/V alerian_Dec2009.pdf>. Acesso em: 23 maio 2010.

OHAYON, M. M. Epidemiology of insomnia: What we know and what we still need to learn. Sleep Medicine Reviews. London, v. 6, n. 2, p. 97-111, abr. 2002.

OXMAN, A. D.; FLOTTORP, S.; HAVELSRUD, K.; FRETHEIM, A.; ODGAARDJENSEN, J.; AUSTVOLL-DAHLGREN, A.; CARLING, C.; PALLESEN, S.; BJORVATN, $B$. A televised, web-based randomised trial of an herbal remedy (valerian) for insomnia. PLoS ONE. San Francisco, v. 2, n. 10, e1040, 2007.

PEARSON, N. J.; JOHNSON, L. L.; NAHIN, R. L. Insomnia, trouble sleeping, and 
complementary and alternative medicine: Analysis of the 2002 national health interview survey data. Archives of Internal Medicine. Chicago, v. 166, p. 1775-1782, 18 set. 2006.

ROTH, T. Insomnia: Definition, prevalence, etiology, and consequences. Journal of Clinical Sleep Medicine. Wetchester, v. 3 (5 supl), s. 7-10, 15 ago. 2007.

SARRAIS, F.; MANGLANO, P. de C. El insomnio. Anales del Sistema Sanitario de Navarra, Pamplona, v. 30, s. 1, p. 121-134, 2007. Disponível em: < http://scielo.isciii.es/pdf/asisna/v30s1/11.pdf>. Acesso em: 23 maio 2010.

SCHULZ, H.; STOLZ, C.; MÜLLER, J. The effect of valerian extract on sleep polygraphy in poor sleepers: a pilot study. Pharmacopsychiatry. Stuttgart, v. 27, n. 4, p. 147-151, jul. 1994.

SMITH, M. T.; PERLIS, M. L.; PARK, A.; SMITH, M. S.; PENNINGTON, J.; GILES, D. E.; BUYSSE, D. J. Comparative meta-analysis of pharmacotherapy and behavior therapy for persistent insomnia. American Journal Psychiatry. Arlington, v. 159, n. 1, p. 5-11, jan. 2002.

STEVINSON, C.; ERNST, E. Valerian for insomnia: a systematic review of randomized clinical trials. Sleep Medicine. Amsterdam, v. 1, n. 2, p. 91-99, 1 abr. 2000.

TAIBI, D. M.; LANDIS, C. A.; PETRY, H.; VITIELLO, M. V. A systematic review of valerian as a sleep aid: Safe but not effective. Sleep Medicine Reviews. London, v. 11, n. 3, p. 209-230, jun. 2007.

TAIBI, D. M.; VITIELLO, M. V.; BARSNESS, S.; ELMER, G. W.; ANDERSON, G. D.; LANDIS, C. A. A randomized clinical trial of valerian fails to improve self-reported, polysomnographic, and actigraphic sleep in older women with insomnia. Sleep Medicine. Amsterdam, v. 10, n. 3, p-319-328, mar. 2009.

TARIQ, S. H.; PULISETTY, S. Pharmacotherapy for insomnia. Clinics in Geriatric Medicine. Philadelphia, v. 24, n. 1, vii, p. 93-105, fev. 2008.

TAYLOR, D. J.; LICHSTEIN, K. L.; DURRENCE, H. H. Insomnia as a health risk factor. Behavioral Sleep Medicine. Philadelphia, v. 1, n. 4, p. 227-247, 2003.

TAYLOR, D. J.; MALLORY, L. J.; LICHSTEIN, K. L.; DURRENCE, H. H.; RIEDEL, B. W.; $\mathrm{BUSH}, \mathrm{A}$. J. Comorbidity of chronic insomnia with medical problems. Sleep. New York, v. 30, n.2, p. 213-218, 1 fev. 2007.

VORBACH, E. U.; GORTELMEYER, R.; BRUNING, J. Therapy of insomnia: The 
efficacy and tolerability of valerian. Psychopharmakotherapie. Sttutgart, v. 3, p. 109-115, 1996.

WALSH, J. K.; ENGELHARDT, C. L. The direct economic costs of insomnia in the United States for 1995. Sleep. New York, 22 supl. 2, s. 386-393, 1 maio 1999.

WORLD HEALTH ORGANIZATION. Monographs on Selected Medicinal Plants. Geneva: World Health Organization, 1999. v. 1, p. 267-275. Disponível em: < http://whqlibdoc.who.int/publications/1999/9241545178.pdf>. Acesso em: 23 maio 2010. 